\title{
ACTIVIDADES UNIVERSITARIAS
}

\section{Universidad de Concepción}

\section{LA ESCUELA DE PEDAGOGIA EN 1925}

URSOS QUE COMPRENDE.-Esla Escuela se fundó en 1919 con el primer año del curso de Inglés. Con posterioridad se crearon sucesivamente el II. III y IV años. hasla que en 1923 quedó completo dicho curso.

Estimulada la Universidad por el éxito alcanzado, procedió a fundar en 1924 el curso de Francés. Para ello envió previamente un profesor a Europa con el fin de que perfeccionara sus conocimientos en Francia, habiendo correspondido esa comisión al señor Luis Arellano, actual profesor del referido curso. Desgraciadamente, apenas creado el primer año, vino la disminución en las entradas de la Universidad y con ella la resolución de las corporaciones directoras universitarias de mantener el curso sólo hasta que terminaran sus estudios los alumnos con quienes se fundó esta nueva sección. Debido a esa circunstancia. el curso de Francés ha funcionado en 1925 sólo con el II año y quedará clausurado en 1927. Iras el funcionamiento del IV àno.

En 1924 se agregó a los cursos arriba nombrados, el de Secrefarios Comerciales.

Finalmente, se acaba de crear en el presente año el Curso de Profesores de Instrucción Primaria.

De modo que en la actualidad funcionan en esta Escuela los 
siguientes cursos: Pedagogia exi Inglés y Francés. Secretariado Comercial y curso Normal para profesores de Instrucción. Primaria.

RAZÓN DE SER DE CADA UNO DE ESOS CURSOS. - Los cursos de Pedagogía en Inglés y Francés llenan doble función: una de carácter profesional o práctico. y otra de naturaleza cultural. La primera, preparando prolesores de Estado en los referidos idiomas, y la segunda, proporcionando. mediante algunos ramos de su plan de estudios, una enseñanza superior humanista.

La utilidad de estos cursos pedagógicos está probada por el hecho de que los alumnos egresados de ellos, casi sin excepción, están ocupados en la enseñanza fiscal y particular en condiciones muy satisfactorias. Por otra parte, ello ha contribuido a aumentar en Concepción y ciudades vecinas el número de cursos privados para la enseñanza del Inglés y Francés, lo que trae ventajas inapreciables para la cultura.

En cuanto a sus funciones culturales, dichos cursos las ejercitan procurando que sus cátedras, como las de Literatura Inglesa. Pedagogia y Psicologia, sirvan también a las personas que deseen hacer esos estudios sin un propósito profesional, y desarrollando otras actividades que como las conferencias, cursos cortos, investigaciones educacionales de problemas locales, etc. fienden a proporcionar oportunidades de mejoramiento cultural a todos los que lo necesiten. También responde a este fin la admisión de alumnos libres, entre los cuales ha contado la Escuela durante el último año a varios profesores de la localidad, a algunas señoritas y jóvenes de otras Escuelas y hasta a profesionales que se dedican a otras carreras distintas de la enseñanza.

- Por lo que hace al curso normal para Prolesores de Instrucción Primaria. su creación responde al ideal, expresado con tanta insistencia y fundamento por las diferentes Asociaciones de maestros del país, de incluir entre las funciones de la Universidad la formación del preceptorado, tal como ocurre hoy 
en Alemania. Italia y otros paises que se preocupan de adaplar la preparación y condiciones del profesorado a las nuevas exigencias de la época. Atribuimos a esta iniciativa una gran importancia. pues ella, junto con elevar la carrera del magisterio. contribuirá, seguramente, a preparar la realización del ideal de la escuela común. Asi lo ha comprendido el señor Direclor de Educación Primaria, quien ha tenido palabras de estimulo para este curso.

\section{LA LABOR DE LOS PROFESORES EGRESADOS DEL CURSO} DE PEDAGOGIA EN INGLÉS.-La calidad de la enseñanza y educación impartida por esta Escuela está probada por los resultados que han oblenido los alumnos egresados de sus aulas y que actualmente dedican sus esfuerzos al cumplimiento de sus altos deberes prolesionales en los diferentes Liceos a que han sido destinados. Dichos resultados no pueden ser más halagadores, ya que los Rectores y Directores respectivos no han tenido sino palabras de aplauso para la actuación sobresaliente de todos nuestros ex-alumnos. De esta manera las influencias del espírilu cientifico y moral de la Universidad de Concepción se difunden día a día en varias ciudades de la República por la vía más noble y segura: la educación.

El amor al trabajo, el anhelo de perfeccionamiento. el espiritu de cooperación social. el patriotismo amplio y bien comprendido. sentimientos a cuyo desarrollo se presta especial atención en la Escuela, se han manifestado no solamente en la labor que esos profesores vienen realizando en sus clases, sino también en las varias iniciativas post-escolares y sociales de que son autores o colaboradores en las diversas poblaciones donde actúan.

MÉTODOS.-Todos los profesores desarrollan su enseñanza guiados por el propósito de formar el espiritu cientifico de los alumnos mediante la adopción de procedimientos de observación. de comparación, de análisis, de inducción. deducción, y por el de reducir en lo posible la transmisión de juicios pre-formados 
a lo estrictamente necesario y sólo para uniformar el criterio de los futuros profesores sobre algunos de los ideales y doctrinas más fundamentales de la educación.

La pasividad del alumno de antes está substituida por la actividad creadora. En lugar de considerar la mente como un receptáculo de capacidad igual para lodos. en el que se arrojan las materias del programa sin preocuparse de si éslas serán elaboradas y aprovechadas, se la considera como función múltiple que debe ensanchar por si misma sus elementos en el medio ambiente.

Esta idea se aplica en la medida de lo posible a todos los cursos, reemplazando las clases de conferencias por la investigación personal, el estudio teórico por el experimento, el trabajo impuesto por el de la actividad espontánea basada en las necesidades actuales.

Mediante éstos y otros medios los profesores educan a los alumnos para la iniciativa. la independencia y la personalidad.

Su preocupación esencial es rodear a los futuros profesores de eslímulos que los impulsen a trabajar siempre buscando la propia superación.

Cultura PEdagógica y Filosófica.-Se presla especial atención a la Pedagogía y Filosofia, como a ciencias de índole fundamental y como a materias bases que han de regir la vida intelectual y moral de los futuros profesores.

Se guian estus cursos en su contenido y métodos por la convicción de que lo esencial en la cultura pedagógica y filosófica del educador es el espíritu profesional y la actitud filosófica. es decir, aquella disposición mental para comprender los valores que encierran el niño y la educación. para afrontar las dificultades de la enseñanza y poder emprender con éxito todas las innovaciones que exige nuestra educación: y, por fin. para que los ideales del Bien. Belleza. Verdad y Justicia sean guía de las acciones y puedan penetrar en la corriente de la vida del profesor como un hálito de pureza ascendente.

La pedagogía moderna, con sus mélodos y toda su compli- 
cación técnica, tiende a un frio intelectualismo donde todo está calculado, los efeclos previstos, las consecuencias compuladas. Hay el peligro de que los profesores que ella forme sean, ante todo, técnicos, investigadores frios, ajenos casi a ese idealismo tan necesario en la educación.

Por otra parte, el deseo muy plausible de reducir el contenido teórico y abstracto en provecho de lo práctico y útil, se va exagerando en forma que ya son muchos los educadores -praclicistas, que miran con un profundo menosprecio las ideas generales, las doctrinas y las teorias. Para ellos, no tienen valor sino los hechos. que los educandos atiendan sólo a las manipulaciones, a la actividad sensorial; que el profesor sepa sólo enseñar, es decir, mantener en orden una clase y meler en las menles adolescentes la práclica, la instrucción de los obreros. comercianles, elc.

Esta manera de pensar y aqueila tendencia de la pedagogia moderna entrañan peligros muy graves para el progreso espiritual. Es necesario evitarlos. esmerándose por la buena preparación filosófica de los futuros profesores. para que mediante ella adquieran la convicción de que la educación no es sólo una técnica más o menos perfecta, sino además una acción abnegada, penetrante e idealista, $y$ de que las ideas y las doctrinas son necesarias para guiar nuestras acciones, produciendo un poco de claridad en la ruta no siempre luminosa de la carrera docente.

QUÉ SIGNIFICA LA CREACIÓN DEL CURSO DE SECRETARIADO COMERCIAL.-Entre las innovaciones llevadas a cabo en esta Escuela merece mención especial la creación del curso de Secretariado Comercial. para aquellos alumnos que, habiendo fracasado en el Curso Pedagógico. deseen utilizar sus estudios de inglés en la consecución de dicha carrera.

El resultado durante el primer año de su funcionamiento ha sido ampliamente satisfactorio, habiendo recibido el tilulo de Secretarios Comerciales trece alumnos.

Los exámenes, rendidos ante una comisión presidida por el 
señor Presidente de la Universidad, pusieron de manifiesto la buena preparación de los alumnos en Redacción Comercial. Taquigrafía y Mecanografia. Estas habilidades y conocimientos unidos a la preparación que tienen los alumnos en. Geografía, Sociología y otros ramos de Humanidades, hará de nuestros Secretarios Comerciales factores valiosos del Comercio. No es de extrañar, entonces, que varios de esos alumnos, al salir con el diploma de la Escuela, hayan ingresado inmediatamente a algunas oficinas y empresa de importancia.

Esta iniciativa tiene un triple significado que bien merece ser conocido: por un lado, es una oportunidad para que las jóvenes egresadas de los Liceos adquieran una carrera segura y digna; por otra parte. facilita el ingreso a las actividades comerciales de elementos que. por su cultura y educación, pueden elevar las condiciones de la vida mercantil hasta hacer de ella. no sólo una actividad de lucro, sino también una función de progreso social en el sentido más amplio. Por último, este ensayo, aunque realizado en forma modesta, es la solución de un grave problema educacional que urge resolver en el pais, consistente en el aprovechamiento de todos aquellos alumnos que, habiendo fracasado parcialmente en un curso, están sin embargo en situación de seguir alguna carrera utilizando la preparación ya adquirida. Medilese en la falange de jóvenes que cada año se retiran de los esfudios superiores con el estigma aplasfante de fracasados, piénsese en el peso peligroso de esa carga para la sociedad, y se comprenderá toda la importancia que toma esta iniciativa ideada por nuestra Universidad.

PENSIONADA. - El Directorio tuvo a bien ampliar el plazo de permanencia de la señorila Corina Vargas en la Universidad de Columbia en Nueva York. EE. UU., hasta Sepliembre de este año. Como se sabe, la señorita Vargas fué enviada por nuestra Universidad a realizar estudio de Psicologia Experimental en aquel prestigioso centro de enseñanza superior.

A su regreso a la Escuela podrá realizar algunos trabajos 
interesantes relacionados con las Ciencias de la Educación y completar sus laboratorios para observaciones psicológicas y pedagógicas.

BIBLIOTECA.-Cuenta con tres mil volúmenes avaluados en $\$ 21,215.00$.

MATERIAL DE ENSEÑANZA.-Dor ahora cuenta la Escuela con un gabinete de Antropometría que ya ha prestado servicios de inestimable valor en el estudio de algunos problemas de importancia local: así, gracias a él. se ha buscado la relación entre el crecimiento de algunos grupos de escolares de niños de Concepción y el factor educación.

Cabe en esta parte hacer notar la falta enorme que hace a la Escuela un Laboratorio de Psicología y cuya adquisición contribuiria no sólo a dar carácter más experimental a los cursos de Pedagogía y Psicologia sino también a mejorar algunos estudios del Curso de Leyes de Concepción. Este Laboratorio nos permitiria tambièn fundar una Oficina de Orientación Profesional que prestaria servicios de gran importancia.

\section{PUBLICACIONES Y COLABORACIÓN A LAS ACTIVIDADES} DE ALGUNOS ESTABLECIMIENTOS DE EDUCACIÓN.-La Escuela. en el empeño de preslar su colaboración a todas las actividades educacionales de la localidad, ha realizado algunos trabajos modestos pero de frascendencia para la orientación pedagógica de algunos planteles de educación. Entre esos trabajos merece mencionarse la aplicación de la escuela de Binet y Simón a la formación de agrupaciones de alumnos en el Liceo de Hombres de Concepción a fin de reemplazar con este sistema cientifico la clasificación de los niños de acuerdo con la estatura.

Con tal motivo. a fin de vulgarizar las ventajas de este sistema entre los educadores de la localidad, la Escuela ha hecho algunas publicaciones como la Ficha Añtropométrica, el Estudio sobre las Pruebas Mentales de Binet. 
PLAN DE FUTURO DESARROLLO.-De acuerdo con el amplio programa que se tiene trazado y en armonia con los propósitos manifestados por las corporaciones directoras de la Universidad al cambiar el nombre de Escuela de Pedagogia por el de Educación, este plantel agregará a los cursos ya existentes una sección especial deslinada a la preparación de maestras infantiles. Con esla inicialiva cree hacer una obra útil, ya que el preparar estas profesionales resuelve el problema de la educación y orientación de los niños menores de 7 años y proporciona una carrera fácil y segura a todas aquellas jóvenes que. habiendo cursado los tres primeros años de Humanidades, desean dedicarse a las tareas educacionales.

El PRESUPUESTO de la Escuela de Pedagogia en 1925. ascendió a $\$ 77.200 .00$, que se distribuyen en esta forma:

I.-Administración.................................. \$ 12.900.00 II.-Personal docente.............................. $58,000.00$ III._Variables (Fomento de la Biblioleca, efc.)...... $\quad 6,300.00$ 